\title{
Intravenous moxifloxacin in routine hospital treatment of respiratory tract infections in China: results of a multicenter, noninterventional study
}

This article was published in the following Dove Press journal:

International Journal of General Medicine

I5 April 201 I

Number of times this article has been viewed

\section{Rongchang Chen' \\ Wenjiang $\mathrm{Ma}^{2}$ \\ Xuezhong $\mathrm{Yu}^{3}$ \\ Xinmin Liu $^{4}$ \\ Jihong Zhu ${ }^{5}$ \\ Hong Liang ${ }^{6}$ \\ Xiaomei $\mathrm{Wu}^{7}$ \\ Tao Guo ${ }^{8}$}

'State Key Laboratory of Respiratory Disease, First Affiliated Hospital of Guangzhou Medical University, China; ${ }^{2}$ Respiratory Department, The First Affiliated Hospital of Medical School of Zhejiang University, China; ${ }^{3}$ Emergency Department, Peking Union Medical College Hospital, China; ${ }^{4}$ Geriatric Department, Peking University First Hospital, China; ${ }^{5}$ Emergency Department, Peking University People's Hospital, China; ${ }^{6}$ Respiratory department, Huadong Hospital Affiliated to Fudan University, China; ${ }^{7}$ Respiratory Department, The Second Affiliated Hospital of Harbin Medical University, China; ${ }^{8}$ Hematology Department, Wuhan Union Hospital, China
Correspondence: Rongchang Chen State Key Laboratory of Respiratory Disease, First Affiliated Hospital of Guangzhou Medical University, China Tel +862083062870

Fax +86 2083062729

Email chenrc@vip.163.com
Objective: To investigate the effectiveness, safety, and tolerability of moxifloxacin (MXF) (intravenous [IV] or sequential therapy [IV followed by oral]) under daily treatment conditions in a large number of patients with respiratory tract infections.

Design: Patients with a diagnosis of respiratory tract infection should be treated with MXF IV and/or tablets $400 \mathrm{mg}$ once daily for a duration at the physician's discretion. For each patient, the physician documented data at an initial visit and at the end of therapy (EOT) visit and/or, in the case of sequential therapy, an interim visit when the patient switched to oral treatment.

Results: A total of 1953 patients treated with MXF were documented and were valid for an effectiveness and safety evaluation. An improvement was observed in 98.1\% ( $n=1911 / 1949)$ of patients treated with MXF. Recovery was documented in $89.9 \%(n=1754 / 1951)$ of the patients. At the EOT visit, severity of infection was assessed to be "relieved" or at least "improved" in $96.5 \%(n=1873 / 1940)$ of the patients. Physicians assessed overall effectiveness as "good" or "very good" in $93.3 \%(\mathrm{n}=1822 / 1953)$ of all patients. The physicians' overall tolerability rating was "very good" or "good" in $93.5 \%(n=1827 / 1953)$ of all patients. The incidence rates of adverse events (AEs) and adverse drug reactions (ADRs) were $0.72 \%(n=14)$ and $0.67 \%$ $(n=13)$, respectively. One serious AE "falling white blood cell count” occurred $(0.05 \%)$, which was also defined as a serious ADR and resolved.

Conclusion: MXF was generally well tolerated and highly effective in the treatment of different respiratory tract infections. The incidence of AEs and ADRs was low. The efficacy, safety, and tolerability information collected in this study confirms the clinical safety profile of MXF and its value as antibiotic treatment for respiratory tract infections.

Keywords: moxifloxacin, respiratory tract infections

\section{Introduction}

Respiratory tract infections, such as community-acquired pneumonia (CAP), acute exacerbation of chronic bronchitis (AECB), or acute bacterial sinusitis, are important causes of morbidity and mortality worldwide. The most frequent causative organism in CAP is Streptococcus pneumoniae or, less commonly, one of the atypical organisms Mycoplasma pneumoniae, Chlamydophila pneumonia, or Legionella pneumophilia. ${ }^{1}$ In AECB, the primary organisms are Haemophilus influenzae, S. pneumonia, and Moraxella catarrhalis. ${ }^{2}$

The antibiotic used to treat any of these respiratory tract infections should be chosen to maximize bacterial eradication and minimize the risk of antimicrobial resistance development. Of particular concern is the emergence of the multidrug-resistant S. pneumonia. ${ }^{3,4}$ 
Moxifloxacin is an 8-metoxifluoroquinolone with a broad spectrum of antibacterial activity. It is effective against Gram-positive pathogens and Gram-negative pathogens, atypical pathogens, and anaerobic bacteria. Among respiratory pathogens, most penicillin- and macrolideresistant and $\beta$-lactamase-producing strains are sensitive to moxifloxacin. ${ }^{5}$

This study was designed to obtain data on the effectiveness and safety of moxifloxacin (administered intravenously [IV] or sequentially [IV followed by oral]) under daily treatment conditions in China in a large number of patients with diagnosed bacterial respiratory tract infections.

\section{Materials and methods Study design}

This was a prospective, noninterventional, noncontrolled, multicenter, postmarketing surveillance trial conducted between February 2006 and March 2007 in China in patients presenting at a hospital as part of a global study. The study was carried out within an approved indication in accordance with US Food and Drug Administration and European Medicines Agency guidelines and applicable local law(s) and regulation(s). Only data and observations surveyed during regular therapy with moxifloxacin were documented. No intervention in the therapeutic decisions of the investigator was allowed. Doctors were instructed to prescribe moxifloxacin for treatment of a respiratory tract infection in the usual manner in accordance with the Chinese marketing authorization as far as possible. No additional diagnostic or monitoring procedures were applied to patients. For each patient, the physician documented data at an initial visit and an end of therapy (EOT) visit and/or, in the case of sequential therapy, an interim visit when the patient switched to oral treatment. Observation parameters were fever, cough, dyspnea, and sputum, which were documented to assess symptom development. Effectiveness parameters included course of severity of infection, course of patient's general condition, course of clinical signs and symptoms, duration until improvement and recovery, and overall assessment of effectiveness by patient and physician. Safety parameters were adverse events (AEs), including adverse drug reactions (ADRs), and global assessment of tolerability.

\section{Data analysis}

Descriptive and explorative analyses were conducted with the software system SAS ${ }^{\circledR}$ for Windows, (Version 9.1; SAS Institute, Lans, NC) According to confidentiality guidelines, each patient was assigned an individual five-digit number (pseudonymization), and only the physicians knew which patient had which number and could identify the patient. The analysis was based on the intent-to-treat (ITT) population. For qualitative variables, absolute and relative frequencies were calculated. For stratified analyses, row percents (row\%) and column percents (col\%) were calculated. For numeric data, the following distribution parameters were calculated: median, mean, standard deviation (SD), minimum, maximum, and $1 \%, 5 \%, 25 \%, 75 \%, 95 \%$, and $99 \%$ percentiles. For coding of verbatim documentation, the following coding systems were applied: concomitant therapies: World Health Organization DD 2005/Q3; adverse events: MedDRA ${ }^{\circledR}$ Version 10.0 .

\section{Study population \\ ITT population/safety population}

The definitions for the safety and the ITT populations were identical in this study. Patients were included in the ITT population if at least one dose of moxifloxacin was taken during the observational period. Seventy-four of 2027 enrolled patients were excluded from ITT analysis due to one or more of the following reasons: i) patient double entry, ii) first visit in more than 2 days before the start of the study (retrospective documentation), and iii) no symptoms at initial visit. Thus, for the ITT population as well as in the safety analysis, data of 1953 patients were analyzed.

\section{Results \\ Demographics}

A total of 1953 patients treated with moxifloxacin were documented and were valid for effectiveness and safety evaluation. The vast majority of patients were of Asian origin (99.8\%); one patient was white $(0.1 \%)$ and one patient was black $(0.1 \%)$. For two patients, information on race was missing $(0.1 \%)$. A total of 1284 patients were male $(65.7 \%)$, and 669 patients were female (34.3\%). The age ranged from 14.0 to 105.0 years with a mean age of $59.6 \pm 17.5$ years. A total of $46.9 \%(n=915 / 1953)$ of the patients suffered from CAP, and $40.1 \%(n=783 / 1953)$ of the patients examined were diagnosed with AECB. Combined infections (CAP and AECB, CAP and other, AECB and other, CAP and AECB and other) were diagnosed in $3.8 \%(n=75 / 1953)$ of all cases, and $15.9 \%(n=310 / 1953)$ of patients were affected by other infections. Most frequent "other infections" were respiratory infection $(7.4 \%, n=23 / 310)$, cross-infection in hospital $(4.8 \%$, $n=15 / 310)$, and inhalation pneumonia $(4.8 \%, n=15 / 310)$. Of the patients, $22.4 \%$ were smokers and $19.3 \%$ past smokers, which is known to be an important etiological factor with 
regard to chronic bronchitis; $57.5 \%$ of the patients were nonsmokers. In terms of alcohol consumption, the majority of patients were abstinent ( $81.0 \%)$. The current infection was rated by the attending physicians to be "mild" in 205 patients (10.5\%), "moderate" in 1217 patients (62.3\%), and "severe", in $518(26.5 \%)$ of the patients. A total of 721 (36.9\%) patients (38.2\% of AECB patients and $30.7 \%$ of the CAP group) had already received at least one antibiotic treatment for the current bacterial infection before the start of moxifloxacin therapy, whereas the majority of 1186 patients $(60.7 \%)$ had not yet received any antibiotic pretreatment (Table 1).

\section{Treatments}

The route of moxifloxacin application was exclusively IV in $78.3 \%$ of patients, whereas moxifloxacin sequential therapy was administered in $21.6 \%$ of the patients. In the case of sequential therapy, 415 out of 421 patients (98.6\%) started with moxifloxacin IV therapy and then switched to oral treatment. A minority of six patients $(1.4 \%)$ changed from

Table I Baseline demographics and diagnosis

\begin{tabular}{|c|c|}
\hline Demographic & Patients $(n=1953)$ \\
\hline \multicolumn{2}{|l|}{ Sex } \\
\hline Male & $1284(65.7 \%)$ \\
\hline Female & 669 (34.3\%) \\
\hline \multicolumn{2}{|l|}{ Age (years) } \\
\hline$<65$ & $1038(53.1 \%)$ \\
\hline$\geq 65$ & $866(44.3 \%)$ \\
\hline Missing & $49(2.5 \%)$ \\
\hline \multicolumn{2}{|l|}{ Diagnosis } \\
\hline Community-acquired pneumonia & 915 (46.9\%) \\
\hline Acute exacerbation of chronic bronchitis & $783(40.1 \%)$ \\
\hline Other infection & $310(15.9 \%)$ \\
\hline Combined infection & $75(3.8 \%)$ \\
\hline \multicolumn{2}{|l|}{ Nicotine consumption } \\
\hline Missing & I 8 (0.9\%) \\
\hline Nonsmoker & I I 22 (57.5\%) \\
\hline Current smoker & $437(22.4 \%)$ \\
\hline Past smoker & $376(19.3 \%)$ \\
\hline \multicolumn{2}{|l|}{ Alcohol consumption } \\
\hline Missing & $17(0.9 \%)$ \\
\hline Abstinent & I58| (8I.0\%) \\
\hline Light & $235(12.0 \%)$ \\
\hline Moderate & $89(4.6 \%)$ \\
\hline Heavy & $31(1.6 \%)$ \\
\hline \multicolumn{2}{|l|}{ Severity } \\
\hline No infection & $4(0.2 \%)$ \\
\hline Mild & $205(10.5 \%)$ \\
\hline Moderate & $1217(62.3 \%)$ \\
\hline Severe & $518(26.5 \%)$ \\
\hline \multicolumn{2}{|l|}{ Antibiotic pretreatment } \\
\hline Missing & $46(2.4 \%)$ \\
\hline No & I I 86 (60.7\%) \\
\hline Yes & 721 (36.9\%) \\
\hline
\end{tabular}

oral to IV treatment. A total of $99.6 \%$ of all patients treated with IV (patients treated exclusively with IV and treated by sequential therapy) received the recommended daily dose of $400 \mathrm{mg}$, six patients $(0.3 \%)$ were administered $800 \mathrm{mg}$ moxifloxacin treatment, and in one case $(0.1 \%)$ information on moxifloxacin IV dose was missing. Oral therapy was administered as a $400 \mathrm{mg}$ daily dose regimen in $99.8 \%$ of the patients, and in only one case $(0.1 \%)$ was information missing.

The mean duration of moxifloxacin treatment was 9.1 days (SD 3.6 days) with a median of 8.0 days. Sequential therapy lasted, on average, 12.5 days (SD 3.8 days). Exclusive IV therapy was applied for, on average, 8.1 days (SD 2.9 days). The total treatment duration varied with the patients' diagnoses: AECB therapy was slightly shorter (8.9 days, SD 3.7 days) compared with CAP therapy (9.2 days, SD 3.4 days) and the therapy for other diagnoses ( 9.0 days, SD 3.7 days), respectively. Combined infections were treated for an average of 8.4 days (SD 2.8 days). The majority of all documented patients treated by sequential therapy $(53.2 \%$, 224/421) received moxifloxacin treatment for a time period of 10-14 days, whereas duration for most patients treated exclusively with IV therapy was 7-9 days (41.2\%, 631/1530). A small proportion of patients was treated for less than 5 days (sequential therapy: 0.5\% ;V therapy: $2.8 \%$ ). A total of $26.8 \%$ treated by sequential therapy and $3.3 \%$ treated by exclusive IV therapy, respectively, were treated for more than 14 days.

\section{Effectiveness results}

At each visit, the patients' condition and severity of infection were assessed and recorded by the attending physician. Additionally, fever and the three most prevalent symptoms (dyspnea, cough, and sputum) were reported (Figure 1). The patients' condition and severity of infection at the start and end of therapy are displayed in Figures 2 and 3.

In total, there were 1949 patients recorded with data on improvement and 1951 patients recorded with data on recovery. A total of $82.5 \%(n=1607 / 1949)$ of the patients had improved after 5 days of treatment; 68.9\% $(n=1345 / 1951)$ patients recovered after 10 days. At the end of the observational period, an improvement was observed in $98.1 \%$ ( $n=1911 / 1949)$ of the respiratory tract infection patients treated with moxifloxacin, and recovery was documented for $89.9 \%(n=1754 / 1951)$ of the participating patients (Figure 4).

Moxifloxacin IV treatment, as well as sequential therapy (moxifloxacin IV/oral), was very effective in the treatment of respiratory tract infections. For $93.3 \%(n=1822 / 1953)$ of the 


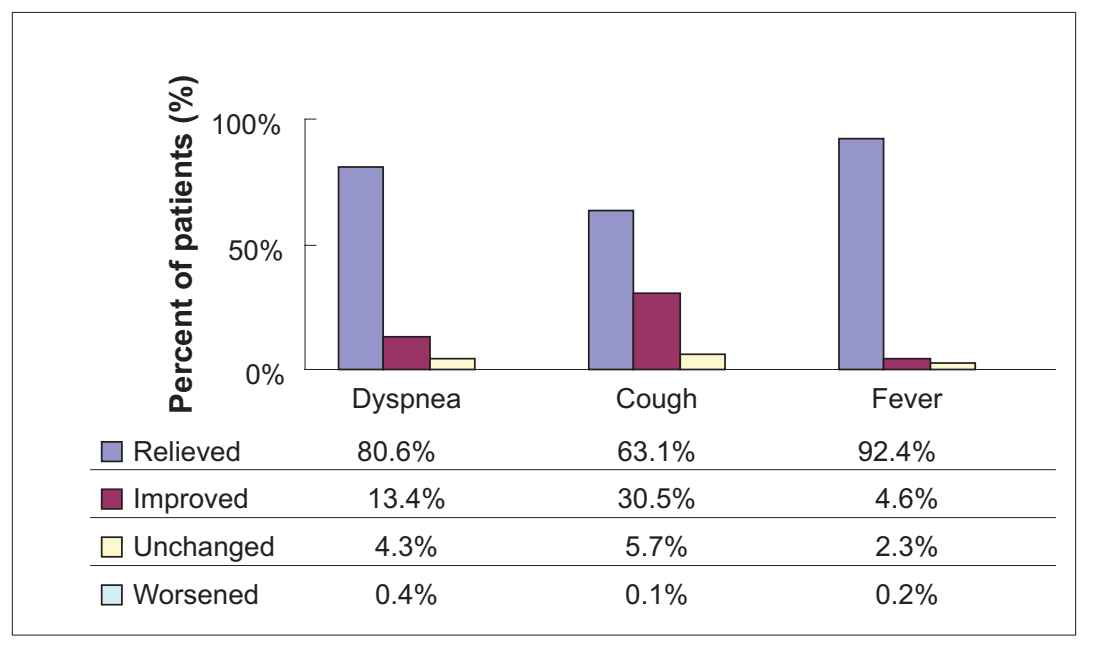

Figure I Course of severity of fever, dyspnea, and cough from first to last visit.

documented patients, effectiveness was rated as "very good" $(56.4 \%, \mathrm{n}=1102 / 1953)$ and "good" $(36.9 \%, \mathrm{n}=720 / 1953)$ by the attending physician. For "sufficient" and "insufficient", $5.3 \%(\mathrm{n}=103 / 1953)$ and $1.3 \%(\mathrm{n}=25 / 1953)$ were rated individually. The overall effectiveness assessment made by the physicians is shown in Figure 5. Stratification by diagnosis revealed best ratings ("very good" and "good") in the patient group suffering from CAP (94.5\%). Of the AECB patients, $92.6 \%$ had "very good" and "good" effectiveness ratings, compared with $88.1 \%$ of patients with other infections. The physicians' final assessment of effectiveness was also stratified by severity of the infection at the initial visit: Effectiveness was assessed as "very good" and "good" in $95.1 \%$ of patients with a "mild" infection, in $94.6 \%$ of patients with a "moderate" infection, and in $89.8 \%$ of patients with a "severe" infection. In multimorbid patients with at least two concomitant diseases, effectiveness was assessed as "very good" and "good" in $91.3 \%$ of patients compared with $95.0 \%$ of patients with fewer than two concomitant diseases.

Physicians recorded that $96.8 \%(n=1890 / 1953)$ of patients were "very satisfied" $(58.2 \%, \mathrm{n}=1137 / 1953)$ or "satisfied" (38.6\%, $n=753 / 1953)$ with the therapeutic effect of moxifloxacin. "Not satisfied" was rated in only $2.9 \%$ $(n=56 / 1953)$ of patients.

\section{Safety and tolerability}

During this open-label moxifloxacin postmarketing surveillance study of 1953 patients in China, moxifloxacin was principally well tolerated. The physicians' overall tolerability rating was "very good" $(57.1 \%, \mathrm{n}=1115 / 1953)$ or "good" $(36.5 \%, \mathrm{n}=712 / 1953)$ in $93.5 \%(\mathrm{n}=1827 / 1953)$ of all patients. The physicians rated tolerability as "sufficient" in $5.1 \%$ and as "insufficient" in $0.8 \%$ of the patients individually (Figure 6).

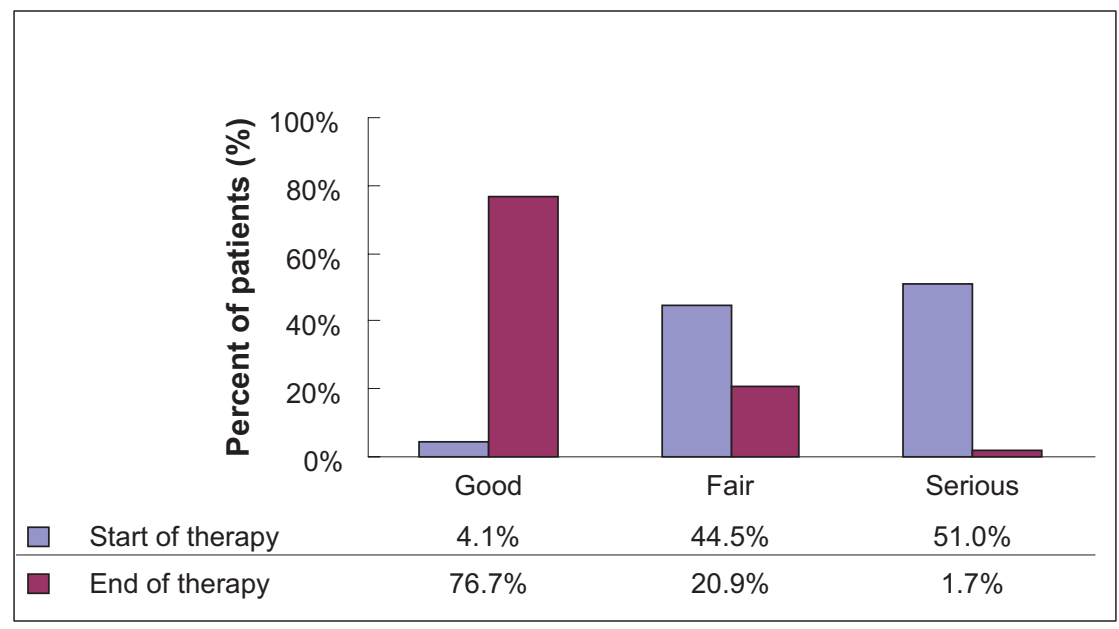

Figure 2 Course of patients' condition from first to last day of therapy. 


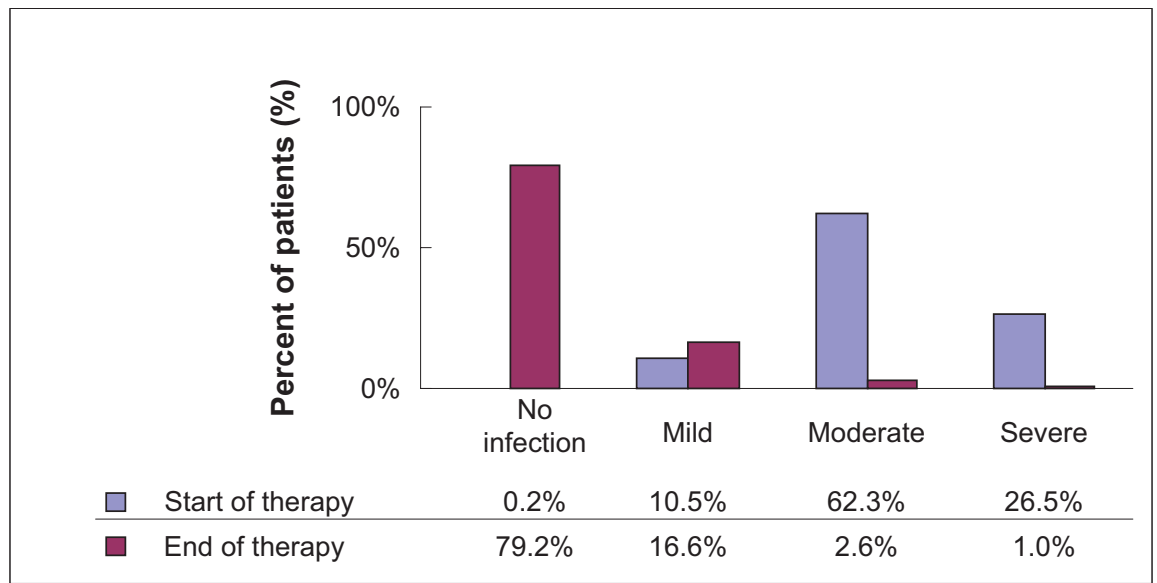

Figure 3 Course of severity of infection from first to last visit.

Pretreatment (95.6\% "very good" or "good") as well as lower morbidity (fewer than two concomitant diseases, 94.4\% "very good" or "good") revealed better results regarding tolerability compared with patients without pretreatment (90.6\% "very good" or "good") and patients with higher morbidity (two or more concomitant diseases, $92.5 \%)$.

The incidence rates of AEs and ADRs were $0.72 \%$ $(\mathrm{n}=14 / 1953)$ and $0.67 \%(\mathrm{n}=13 / 1953)$, respectively. Symptoms such as nausea and vomiting, which are well known in antibiotic treatment and also with quinolones, were predominant (each with $0.15 \%$ ). One serious AE occurred $(0.05 \%)$, which was also defined as a serious ADR. In this case, an 82-year-old male patient encountered "falling white blood cell count", which led to "hospitalization necessary or prolonged", but the event finally resolved.

In total, there were 33 AEs. Out of those, 29 were considered ADRs. Twenty-eight of the AEs (24 of 29 ADRs) were resolved or improved by the end of the observational period. In other cases, information on event outcome was missing. Table 2 summarizes AEs and ADRs.

\section{Discussion}

Infections occur in the respiratory tract more frequently than in any other site. Acute respiratory tract infections are the most common illnesses in humans and are associated with significant morbidity and mortality. Antibiotics are commonly prescribed empirically for respiratory tract infections in adults and children in primary care.

The inappropriate prescribing of antibiotics has the potential to cause drug-related AEs, escalate the prevalence of antibiotic-resistant organisms in the community, and increase primary care consultation rates for minor illness (Standing Medical Advisory Committee 1998). How to use antibiotics in the treatment of respiratory tract infection appropriately is a big challenge for prescribers. Fortunately, there are national and international guidelines for the treatment of respiratory tract infections, eg, the

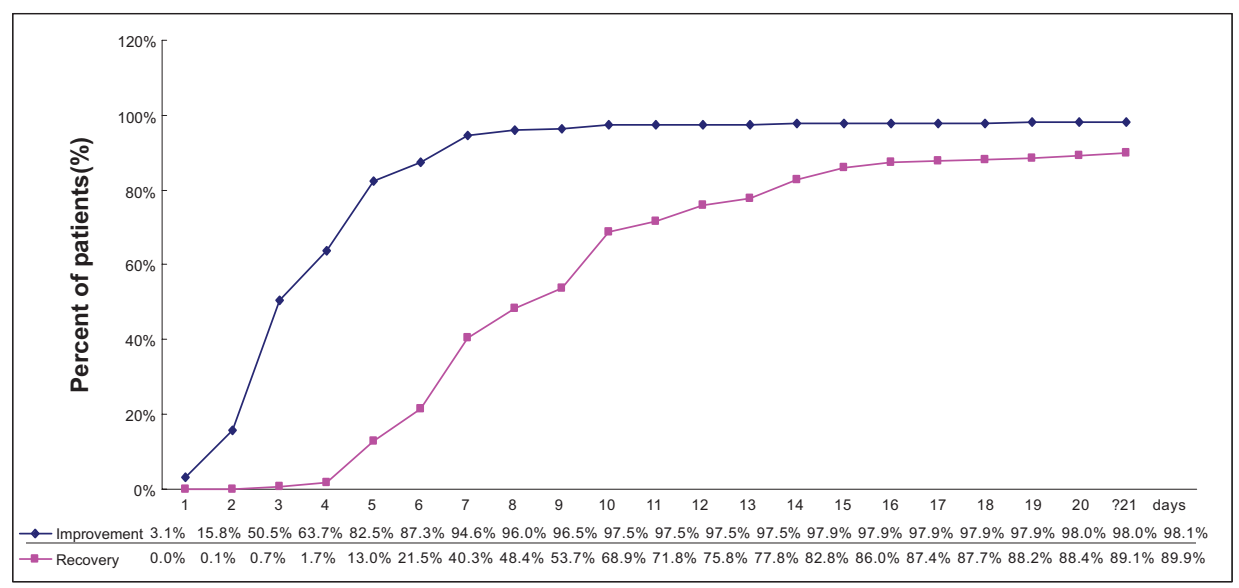

Figure 4 Duration until improvement and recovery after first administration of moxifloxacin. 


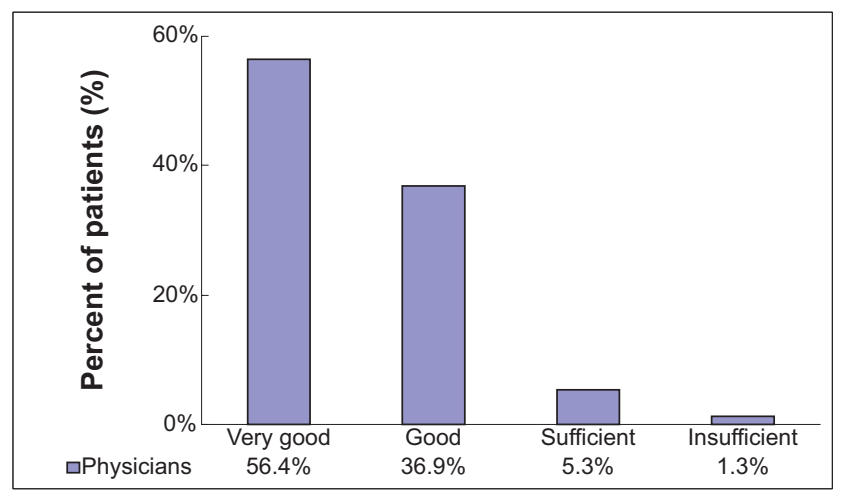

Figure 5 Physicians' final assessment of effectiveness.

European Respiratory Society's lower respiratory tract infections guidelines. ${ }^{6}$ In China, guidelines were also established to guarantee the appropriate use of antibiotics in respiratory tract infection patients, especially CAP and AECB patients. Moxifloxacin is recommended in both Chinese guidelines ${ }^{7,8}$ for treating CAP and AECB. For CAP, moxifloxacin is recommended for preliminary empirical use in the following patients: young to middleaged adults without underlying disease, elderly people or those with underlying disease, patients who have been hospitalized but not admitted to the intensive care unit (ICU), those using a combination with other antibacterials in ICU for Pseudomonas aeruginosa infection risk, and those with aspiration factor. For AECB, moxifloxacin is recommended for empirical use in Grade III and IV patients without $P$. aeruginosa infection risk. More and more physicians have experience in using moxifloxacin in China; nevertheless, there is still the need to collect and analyze local data, especially with a large sample size in clinical practice, in order to gather the newest efficacy data in treatment with moxifloxacin.

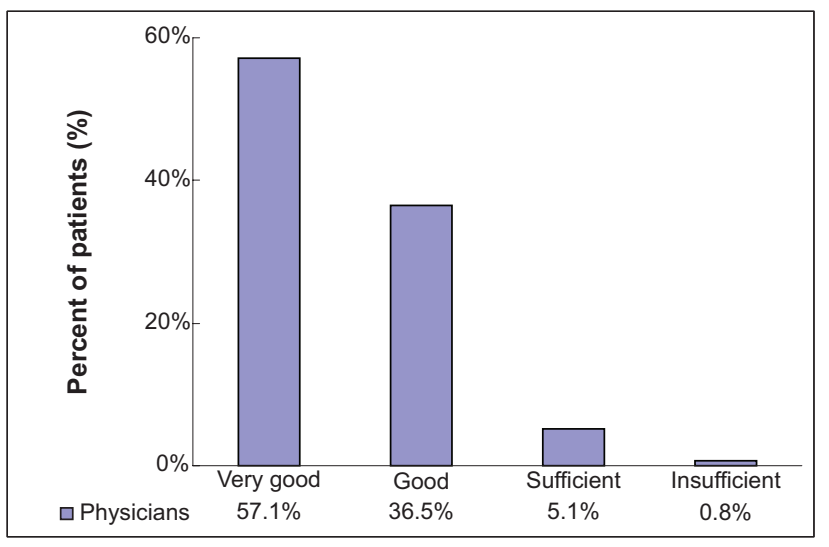

Figure 6 Physicians' final assessment of tolerability.
Table 2 Incidence of adverse events (AEs) and adverse drug reactions (ADRs) by MedDRA system organ classes and preferred terms

\begin{tabular}{|c|c|c|}
\hline \multirow{3}{*}{$\begin{array}{l}\text { System organ classes } \\
\text { and preferred terms }\end{array}$} & \multicolumn{2}{|c|}{ Patients $(n=1953)$} \\
\hline & $\overline{\mathrm{AE}}$ & ADR \\
\hline & n (\%) & n (\%) \\
\hline Patients with AE/ADR & $14(0.72)$ & $13(0.67)$ \\
\hline Patients without $A E / A D R$ & 1939 (99.28) & $1940(99.33)$ \\
\hline Gastrointestinal disorders & $3(0.15)$ & $3(0.15)$ \\
\hline $\begin{array}{l}\text { General disorders and } \\
\text { administration site conditions }\end{array}$ & $2(0.10)$ & I (0.05) \\
\hline Hepatobiliary disorders & I (0.05) & I (0.05) \\
\hline Investigations & $2(0.10)$ & $2(0.10)$ \\
\hline Metabolism and nutrition disorders & $3(0.15)$ & $2(0.10)$ \\
\hline Nervous system disorders & $2(0.10)$ & I (0.05) \\
\hline Psychiatric disorders & $3(0.15)$ & $3(0.15)$ \\
\hline Skin and subcutaneous tissue disorders & $4(0.20)$ & $4(0.20)$ \\
\hline Vascular disorders & $2(0.10)$ & $2(0.10)$ \\
\hline
\end{tabular}

In our study, 2027 subjects were enrolled and 1953 were included in the statistical analysis. The mean age of all patients was 59.6 years (SD 17.5 years). A patient age over 45 years should be considered a major factor for defining the presence of chronic bronchitis. Indeed, AECB patients were more frequently older patients $(80.8 \%$ of them were aged 50 years and older), whereas CAP patients were nearly evenly distributed in the age range of 30-79 years.

The overall effectiveness assessment showed that for 1822 (93.3\%) of 1953 documented patients, effectiveness was rated as "very good" and "good" by the attending physician. Liu and Landen reported in 2007 that in their postmarketing surveillance of moxifloxacin in treating respiratory tract infection, improvement was observed in $69.1 \%$ of the patients after 3 days of moxifloxacin treatment and in $90.4 \%$ after 5 days. ${ }^{9}$ Full recovery had occurred in $71.3 \%$ by 7 days and in $86.8 \%$ by 10 days. Physicians rated moxifloxacin as "very good" or "good" in $92 \%$ of patients for effectiveness. The results are similar in both studies, which demonstrated moxifloxacin to be of good effectiveness in treating respiratory tract infection. ${ }^{9}$

For safety consideration, moxifloxacin treatment was well tolerated, with physicians rating overall tolerability as "very good" and "good" in $93.5 \%$ of patients. The physicians rated tolerability to be "sufficient" in $5.1 \%$ and to be "insufficient" in $0.8 \%$ of the patients. Pretreatment (95.6\%) as well as lower morbidity (fewer than two concomitant diseases, 94.4\%) corresponded to better results regarding tolerability compared with "no" pretreatment $(90.6 \%)$ and higher morbidity (two or more concomitant diseases, 92.5\%). The incidence rates of AEs and ADRs were $0.72 \%$ and $0.67 \%$, 
respectively. Symptoms such as nausea and vomiting, which are well known in antibiotic treatment, were predominant $(0.15 \%$, respectively). These low numbers underline the very good tolerability and safety of moxifloxacin. Similar results were acquired from Liu and Landen's study. ${ }^{9}$ Physicians rated moxifloxacin as "very good" or "good" in $90.8 \%$ of the patients for tolerability. A total of 129 AEs occurred in 74 (1.9\%) patients and mainly involved either the gastrointestinal or nervous system. All events were mild or moderate, and most resolved or improved after stopping treatment. ${ }^{9}$

This study involved 150 investigators and enrolled a large number of patients in China. The results are helpful for physicians in clinical practice for treating AECB. Although this study lacked bacteriological testing and did not evaluate bacterial outcome, in medical practice, antibiotics are evaluated on the basis of clinical signs and symptoms. Nevertheless, this study is a single-arm, openlabel study, and bias is inevitable when evaluating final effectiveness. Stricter studies in clinical practice are needed to further investigate this area. In general, the data of this study provide a large sample of evidence from China for rational antibiotic usage in treating respiratory tract infections in the future.

\section{Conclusion}

In this observational study, moxifloxacin was generally well tolerated and highly effective in the treatment of different respiratory tract infections requiring initial IV therapy. The incidence of AEs and ADRs was low. The safety and tolerability information collected in this study confirms the clinical safety profile of moxifloxacin and its benefit as a valuable choice of antibiotic treatment for respiratory tract infections.

\section{Acknowledgment/Disclosure}

Bayer Healthcare Company Ltd provided funding for this study and for preparation of the manuscript.

\section{References}

1. Woodhead M. Community-acquired pneumonia in Europe: causative pathogens and resistance patterns. Eur Respir J Suppl. 2002;36: $20 \mathrm{~s}-27 \mathrm{~s}$.

2. American Thoracic Society, European Respiratory Society. Standards for the Diagnosis and Management of Patients with COPD. http://www. thoracic.org/clinical/copd-guidelines/resources/copddoc.pdf. Accessed January 13, 2011.

3. Scheld WM. Maintaining fluoroquinolones class effectiveness: review of influencing factors. Emerg Infect Dis. 2003;9:1-9.

4. Lonks JR, Garau J, Medeiros AA. Implications of antimicrobial resistance in the empirical treatment of community-acquired respiratory tract infections: the case of macrolides. J Antimicrob Chemother. 2002; 50(Suppl S2):87-92.

5. Blondeau JM. A review of the comparative in vitro activities of 12 antimicrobial agents, with a focus on five new respiratory quinolones. J Antimicrob Chemother. 1999;43(Suppl B):1-11.

6. Woodhead M, Blasi F, Ewig S, et al. Guidelines for the management of adult lower respiratory tract infections. Eur Respir J. 2005; 26:1138-1180.

7. Liu Y. Diagnosis and treatment guideline for community-acquired pneumonia. Chin J Tuberc Respir Dis. 2006;29:654.

8. Yao W. Diagnosis and treatment guideline for chronic obstructive pulmonary disease. Chin J Tuberc Respir Dis. 2007;30:15.

9. Liu LY, Landen H. Treatment of respiratory tract infections with moxifloxacin: results of postmarketing surveillance in China. Int J Clin Pract. 2007;61:1509-1515.
International Journal of General Medicine

\section{Publish your work in this journal}

The International Journal of General Medicine is an international, peer-reviewed open-access journal that focuses on general and internal medicine, pathogenesis, epidemiology, diagnosis, monitoring and treatment protocols. The journal is characterized by the rapid reporting of reviews, original research and clinical studies across all disease areas.

\section{Dovepress}

A key focus is the elucidation of disease processes and management protocols resulting in improved outcomes for the patient.The manuscript management system is completely online and includes a very quick and fair peer-review system. Visit http://www.dovepress.com/ testimonials.php to read real quotes from published authors. 